\title{
Evaluation Des Attaques De Termites (Isoptera Brulle, 1832) Sur Quatre Essences Forestieres D'importance Economique Au Togo (Afrique De L'ouest)
}

\author{
Jean Norbert B.K. Gbenyedji \\ Boris Dodji Kasseney \\ Seth Wolali Nyamador \\ Banibéa Bassan Sanbena
}

Laboratoire d'Entomologie Appliquée,

Département de Zoologie et de Biologie Animale, Université de Lomé, Togo

Adzo Dzifa Kokutsè

Kouami Kokou

Laboratoire de Botanique et d'Ecologie végétale,

Faculté des sciences, Université de Lomé, Togo

Isabelle Adolé Glitho

Laboratoire d'Entomologie Appliquée,

Département de Zoologie et de Biologie Animale, Université de Lomé, Togo

doi: 10.19044/esj.2016.v12n9p333 ～URL:http://dx.doi.org/10.19044/esj.2016.v12n9p333

\begin{abstract}
Termites are one of the most abundant invertebrates in tropical ecosystems, where they play a major ecological role in litter decomposition. Despite their important role, termites can cause considerable damage to wood structures in most tropical countries. The aims of this study was to evaluate termite attack in four economically important species in Togo including Antiaris africana Engl (Moraceae), Pterocarpus erinaceus Poir (Fabaceae), Triplochiton scleroxylon Schum (Malvaceae) (all local species) and teak or Tectona grandis Linné (Lamiaceae). For the latter species, termites were infested on $83.49 \%$ of 630 feet examined. However, the rate and type attacks differ from one plantation to another. On the studied forest species, termites were collected on about $97 \%$ of Triplochiton scleroxylon feet. For this species, at least $31 \%$ of examined feet have had their wood attacked. For the other two species, Pterocarpus erinaceus and Antiaris africana respectively about $80 \%$ and $67 \%$ of examined feet were attacked by termites. Amitermes evuncifer, Ancistrotermes guineensis and Microtermes lepidus were species of termite often harvested from trees examined. However the last two species namely A. guineensis and M. lepidus are
\end{abstract}


encountered on the bark of trees inside veneers unlike A. evuncifer harvested in the heart of the wood. Therefore, the latter species deserves special attention as likely to cause serious damage not only to these species but also other species not taken into account in this study. It is therefore necessary to consider adequate control methods against these species.

Keywords: Damage, Termites, Amitermes evuncifer, Forest species, Togo

\section{Résumé}

Les termites constituent l'un des groupes d'invertébrés les plus abondants dans les écosystèmes tropicaux, où ils jouent un rôle écologique majeur dans la décomposition des litières. En dépit de leur important rôle, les termites peuvent causer des dommages considérables aux structures en bois dans la plupart des pays tropicaux. Le but de cette étude était d'évaluer les attaques des termites sur quatre essences économiquement importantes du Togo que sont Antiaris africana Engl (Moraceae), Pterocarpus erinaceus Poir (Fabaceae), Triplochiton scleroxylon Schum (Malvaceae) et le teck ou Tectona grandis Linné (Lamiaceae). Pour cette dernière espèce, les termites ont infesté $83,49 \%$ des 630 pieds examinés. Néanmoins, les taux et le type des attaques diffèrent d'une plantation à une autre. Concernant les essences forestières étudiées, les termites ont été récoltés sur environ $97 \%$ des pieds de Triplochiton scleroxylon. Pour cette espèce, au moins 31\% des pieds examinés ont eu leurs bois attaqués. Pour les deux autres espèces, Antiaris africana et Pterocarpus erinaceus, respectivement environ 80 et $67 \%$ des pieds examinés ont été infestés par les termites. Amitermes evuncifer, Ancistrotermes guineensis et Microtermes lepidus ont été les espèces de termite fréquemment récoltées sur les arbres examinés. Toutefois les deux dernières espèces à savoir $A$. guineensis et $M$. lepidus ne sont rencontrées que sur l'écorce des arbres à l'intérieur des placages contrairement à $A$. evuncifer récoltée au cœur du bois. De ce fait, cette dernière espèce mérite une attention particulière car susceptible de causer de sérieux dégâts non seulement à ces différentes espèces mais également à d'autres espèces non prises en compte dans cette étude. Il s'avère donc nécessaire d'envisager des méthodes de contrôle adéquates contre ces espèces.

Mots clés : Dégâts, Termites, Amitermes evuncifer, Essences forestières, Togo

\section{Introduction}

Les termites sont des insectes xylophages et consommateurs de bois morts. Ils sont à l'origine des principaux dégâts observés sur le bois ouvré (Anani Kotoklo et al., 2010). De ce fait, ils sont redoutés à l'échelle 
mondiale pour la gravité et la variété de leurs attaques. Selon Logan et al. (1990) près de $10 \%$ des espèces de termites décrites sont reconnues comme nuisibles aux végétaux vivants et aux bâtiments. Depuis quelques décennies, une attention particulière est portée à l'étude des attaques des termites, à cause de leur intérêt économique. Un autre aspect destructeur des termites, relativement peu étudié en Afrique, et plus particulièrement au Togo, est l'attaque des végétaux vivants. Sur cet aspect, quelques études ont été réalisées au Togo sur les arbres dans les périmètres du Campus universitaire de Lomé (Anani Kotoklo et al., 2010), sur le teck (Gbenyedji, 2009) et sur les plantations de canne à sucre (Anani Kotoklo, 2007). Ces différents travaux ont montré que les taux d'attaques des végétaux vivants peuvent atteindre et même dépasser les $60 \%$ (cas des cannes à sucre et du teck). Il ressort de ces mêmes travaux que les termites peuvent endommager et tuer les arbres qu'ils attaquent.

Le Togo n'est pas un pays forestier mais l'exploitation de bois de quelques reliques de forêts contribue à l'amélioration du Produit Intérieur Brut (PIB). Thiam (1991) a estimé à 100 milliards FCFA, soit 10\%, la contribution annuelle du secteur forestier au PIB du Togo si l'on prend en compte l'ensemble des filières d'exploitation (exploitation du bois, pharmacopée, confection des éponges, cure-dents, cueillette de fruits et légumes sauvages). En 2007, le Togo a exporté près de $3500 \mathrm{~m}^{3}$ de madriers d'espèces de valeur commerciale (essentiellement du Pterocarpus erinaceus) dont seulement $500 \mathrm{~m}^{3}$ ont été exploités légalement (OIBT, 2008). Selon les statistiques, entre août et septembre 2008, environ 26 conteneurs de $42,25 \mathrm{~m}^{3}$ de madriers et 10 d'équarries de Pterocarpus erinaceus ont transité par le port de Lomé (Ali, 2009).

Sur le plan mondial, on estime que les teckeraies couvrent 5,7 millions d'ha. En Afrique tropicale, elles font près de 250000 ha dont 16000 ha au Togo (Ganglo, 1999). La production mondiale de bois de teck (environ 2 millions de $\mathrm{m}^{3}$ par an) couvre actuellement $1 \%$ des besoins mondiaux en bois d'œuvre. C'est l'essence la plus recherchée. En Afrique, le plus gros exportateur est la Côte d'Ivoire avec plus de $100000 \mathrm{~m}^{3}$ de grumes par an (127000 $\mathrm{m}^{3}$ en 1998), suivi de loin par le Ghana, le Nigeria et le Togo (1700 $\mathrm{m}^{3}$ au cours des 4 premiers mois de 2004) selon Louppe (2005). Les grumes de gros diamètre provenant des forêts naturelles peuvent atteindre des prix très élevés. En 1998 la valeur des grumes de tranchage étaient de 1700-2000 $\mathrm{US} \$ / \mathrm{m}^{3}$ ), ce qui fait du teck le bois de luxe par excellence (Rance et Monteuuis, 2004). En Afrique, les plantations de teck ne semblent pas avoir de maladies graves à l'exception des pourridiés qui peuvent causer des dégâts importants, notamment dans les plantations du sud de la Côte d'Ivoire, du Bénin et Togo mais aussi au Nigeria et en Tanzanie (Bhat, 2000). Toutefois les travaux réalisés par Gbenyedji (2009), montrent que les 
termites sont responsables des dégâts sur les tecks et par conséquent diminue la valeur marchande de son bois. En plus de tecks, les attaques de termites sont également signalées sur d'autres essences forestières par les exploitants de bois. Les quelques unes de ces essences feront aussi l'objet de cette étude.

Compte tenu de l'intérêt économique de ces forêts, il est donc important de répertorier les espèces de termites ravageurs de ces ligneux et d'évaluer leurs impacts dans la perspective d'un développement de méthodes de contrôle adéquat.

\section{Matériel et méthodes}

\section{Sites d'étude}

L'étude a été menée dans les peuplements de Pterocarpus erinaceus localisés dans la savane boisée de la Réserve de Faune de Togodo (RFT) et des peuplements d'Antiaris africana et de Triplochiton scleroxylon localisés dans la formation semi-décidue de la même réserve. La RFT est localisée au sud Est du Togo, entre $1^{\circ} 20^{\prime}$ et $1^{\circ} 40^{\prime}$ de longitude Est et entre $6^{\circ} 40^{\prime}$ et $6^{\circ} 50^{\prime}$ de latitude Nord. En ce qui concerne les tecks, l'évaluation des attaques a été faite dans 07 plantations de différents âges situées dans la même zone climatique (Figure 1). Le climat est subéquatorial. La moyenne annuelle des précipitations est de 1000 à $1200 \mathrm{~mm}$ et la température moyenne annuelle est voisine de $27^{\circ} \mathrm{C}$. Les essences forestières dominantes dans la RFT sont des Combrétacées (Anogeissus sp.), Bombacacées (Ceiba spp.), Sterculiacées (Cola cordifolia), Césalpiniacées (Afzalia africana) et Euphorbiacées (Uapaca togensis). Dans les plantations, en plus de tecks (Tectona grandis), on retrouve des espèces dont les plus fréquentes sont Panicum maximum, Eupatorium sp., Abrus precatorius, Tephrosia vogelii... Les sols développés dans la région appartiennent à trois classes de sols selon la classification pédologique de l'IRD (Institut de Recherche Développement) décrite par Aubert en 1965 (FAO/PNUE, 1972; Adjonou, 2011). Ce sont des vertisols (argiles noires tropicales), les sols ferrugineux tropicaux, et des sols d'apports, modaux sur alluvions fluviales. 


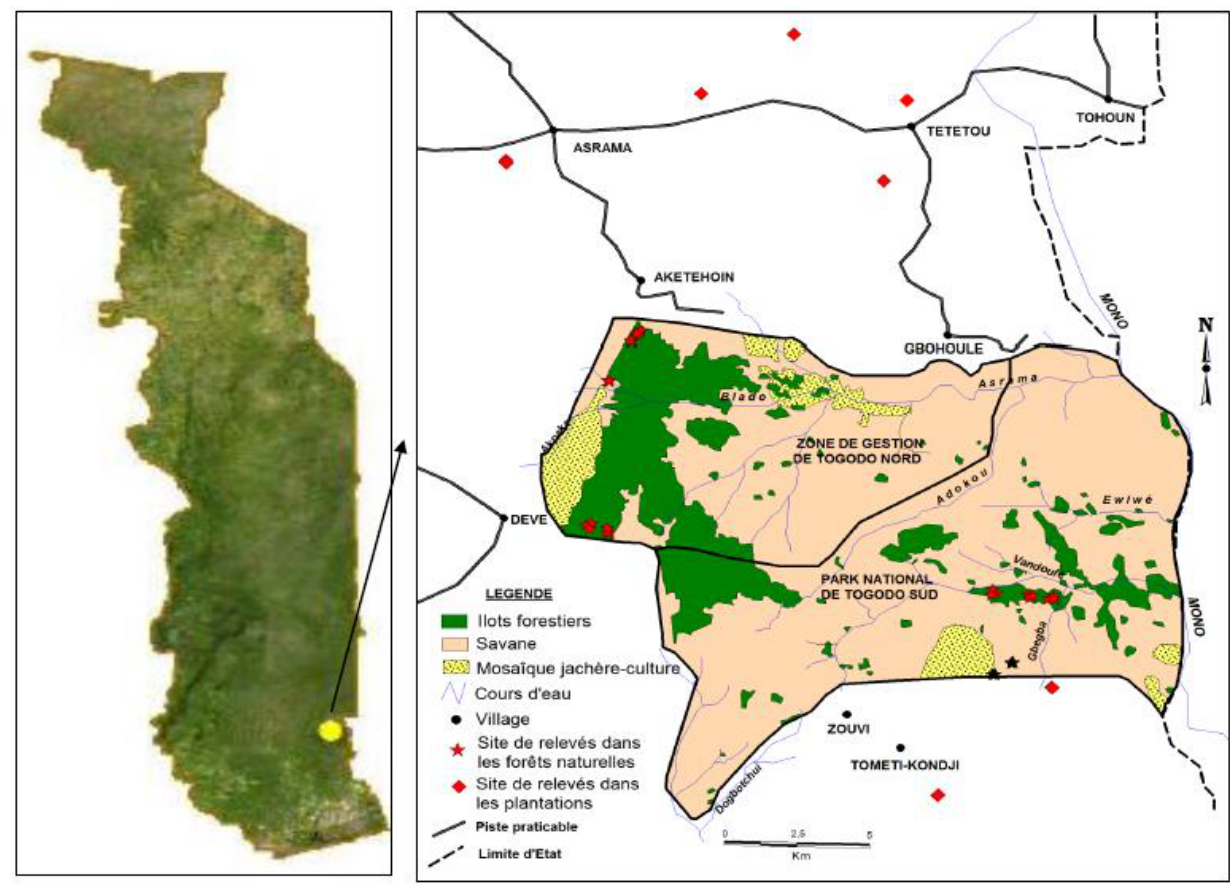

Figure 1 : Carte montrant la zone de l'étude

\section{Matériel végétal}

Quatre essences ont été retenues pour cette étude. Il s'agit de Pterocarpus erinaceus Poir. (Fabaceae), Antiaris africana Engel (Moraceae), Triplochiton scleroxylon K. Schum (Malvaceae) et Tectona grandis Linné (Lamiaceae).

Le choix de ces 4 essences se justifie par leur valeur économique car ce sont des espèces dont le bois est exploité et exporté.

\section{Méthode d'évaluation des attaques de termites}

Dans les plantations de tecks

Sept plantations de tecks d'âges différents $(0,2,4,6,8,10$ et 12 ans) et d'un hectare chacune ont fait l'objet de notre étude. Le choix de ces plantations est fait dans le but de comparer les degrés d'infestation en fonction de l'âge des arbres. Dans chacune des plantations un transect de 100 m x $5 \mathrm{~m}$ a été délimité. Dans chaque transect, au moins 90 pieds de tecks ont été observés en suivant de façon systématique le transect dans une direction nord-sud. Le tronc de chaque pied est examiné sur une hauteur 2,5 m à partir $\mathrm{du}$ sol à la recherche de termites et des signes de leur présence (structures biogéniques). Les termites sont également recherchés dans le sol aux alentours immédiats des pieds prospectés. Les termites récoltés sont conservés dans l'alcool $96^{\circ}$. 


\section{Dans la forêt}

Dans les peuplements des 3 essences forestières retenues ( $P$. erinaceus, $A$. africana et $T$. scleroxylon), la même méthode a été appliquée. Les pieds des essences forestières ont été examinés de façon similaire que les tecks.

\section{Caractérisation des attaques}

Les infestations ont été classées en 5 types selon une échelle de cotation ci-après (Tableau 1).

Tableau 1 : Echelle de cotation des infestations de termites sur les arbres.

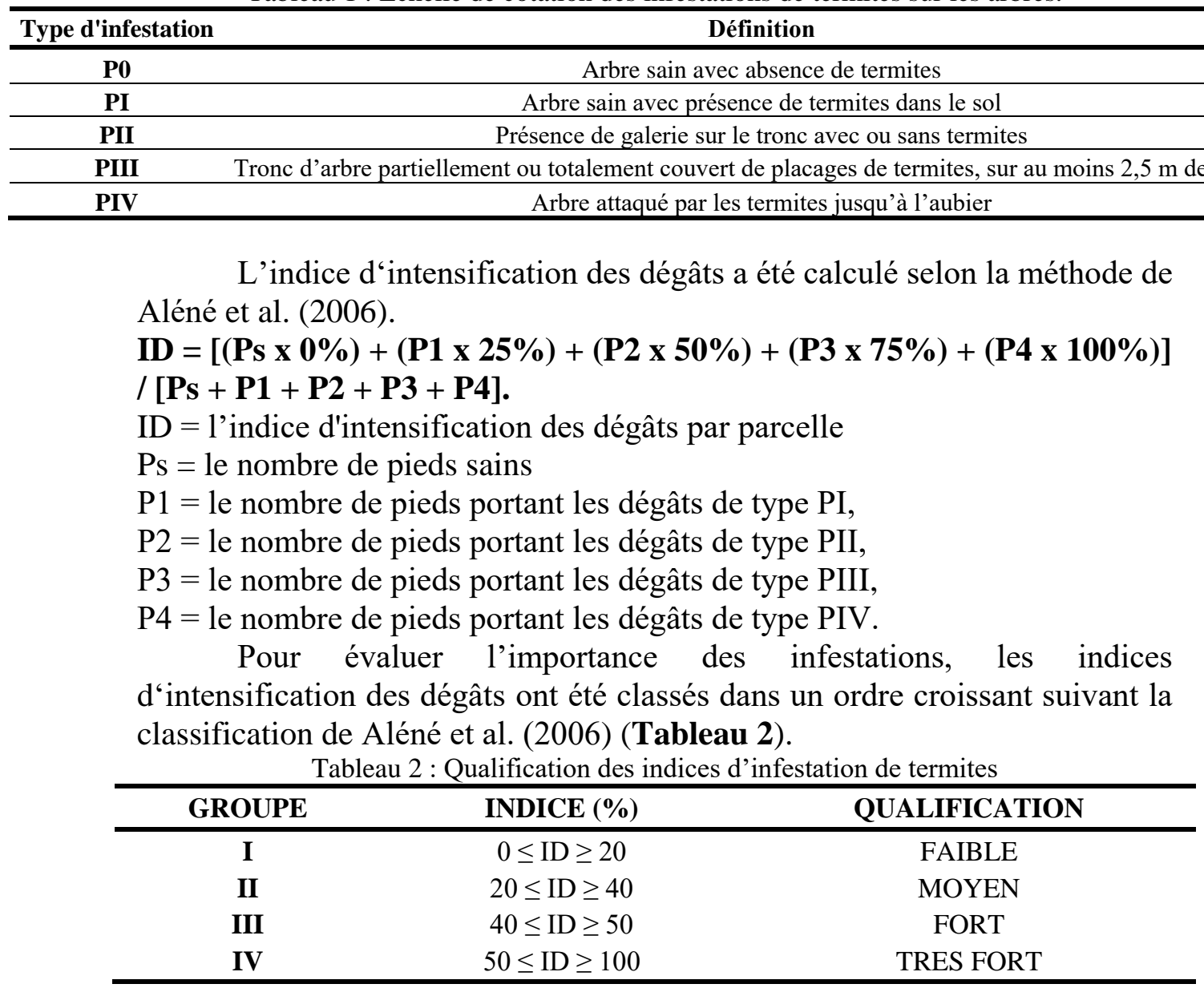

\section{Calcul du taux d'attaque}

Le taux d'attaque des essences par les termites dans chaque peuplement a été calculé selon la formule suivante :

$$
T i=\frac{N P i}{N P t} \times 100
$$


avec,

Ti $=$ Taux d'attaque

$\mathbf{N P i}=$ Nombre de pieds portant les attaques de termites

$\mathbf{N P t}=$ Nombre total de pieds observés dans chaque peuplement

\section{Analyse des données}

Les données obtenues ont été soumises à une analyse de variance (ANOVA) et comparées avec le test multi variable de Newman-Keuls au seuil de 5\% avec le logiciel SPSS, version 17.0.

\section{Résultats}

\section{Espèces de termites récoltées sur les arbres}

Un total de 11 espèces a été récolté sur l'ensemble des sites prospectés. Il s'agit de: Ancistrotermes guineensis, Macrotermes subhyalinus, Microtermes lepidus, Pseudacanthotermes militaris, Odontotermes aff. erraticus, Amitermes evuncifer, Microcerotermes parvus, Fulleritermes tenebricus, Nasutitermes arborum, Adaiphrotermes sp. et Basidentitermes potens. Ces deux dernières espèces ont été récoltées dans le sous sol aux alentours immédiats des arbres examinés.

\section{Analyse quantitative des attaques des termites Plantations de tecks}

Un total de 630 pieds de tecks a été examiné. Sur cet ensemble, $83,49 \%$ des pieds ont présenté des attaques de termites. Cependant, les taux d'attaque diffèrent d'une plantation à une autre.

Dans la plantation de 0 an, le taux moyen d'attaque s'élève alors à $43,33 \pm 3,6 \%$ (Figure 2). Sur les 43,33\% des pieds infestés, nous avons observé 33,33\% de pieds apparemment sains à la surface (Figure 3) dont le sous-sol comporte le système racinaire infesté par les termites (PI de l'échelle de cotation). Les galeries et des placages sur le tronc occupent $8,89 \%$ des plantes alors qu'un seul plant de teck de 0 an $(1,11 \%)$ a été complètement détruit par les termites (Figure 4).

Dans la plantation de tecks de 2 ans, le taux moyen d'infestation est de $83,34 \pm 3,5 \%$ (Figure 2). Ces infestations se situent essentiellement sur la présence des galeries $(64,44 \%)$, des placages $(7,78 \%)$. Le pourcentage de pieds présentant des attaques sur l'écorce et dans le bois a été évalué à 3,33\% (PIV de l'échelle de cotation) (Figure 3).

Pour ce qui est de la plantation de tecks de 4 ans, nous avons enregistré un taux d'infestation de $86,66 \pm 3,1 \%$ (Figure 2) dont 3,33\% de pieds sains avec des termites sous le système racinaire, $81,11 \%$ de pieds avec des galeries sur le tronc et $2,22 \%$ de pieds ayant le bois attaqué. Dans cette 
plantation en revanche nous n'avons pas observé de placage sur les troncs (Figure 3).

Tous les pieds examinés dans la plantation de tecks de 6 ans ont été tous infestés avec 63,34\% d'infestation de type PII ; 32,22\% d'infestation de type PIII et 4,44\% d'attaques de type PIV (Figure 3). Par ailleurs, les tecks de 8 ans ont enregistré un taux moyen d'infestation de 97,78 $\pm 0,7 \%$ dont essentiellement $70,00 \%$ d'infestation de type PII, $14,44 \%$ de type PIII et 5,56\% d'attaque de type PIV. Dans les plantations de tecks de 10 ans, tous les pieds ont été infestés avec 77,78\% de types PII, 14,44\% d'attaques de type PIII et 7,78\% de type PIV. Enfin dans la plantation de tecks de 12 ans, le taux moyen d'infestation est de $73 \pm 1,2 \%$. Les termites sont observés sur les racines (infestation de type PI) avec 12,22\%, sur la présence des galeries $(48,89 \%)$, des placages $(8,89 \%)$ et de $3,33 \%$ d'attaques de l'écorce et dans le bois. $26,67 \%$ des pieds sont sains (P0 de 1'échelle de cotation).

En somme, de l'analyse des résultats, le type d'attaque PIV comporte numériquement très peu d'arbres. Tous les pieds appartenant à ces niveaux présentent des attaques directes des termites sur le bois. Ce type d'attaques diffère et augmente selon les âges des plantations sauf dans la plantation de 12 ans où nous avons observé une diminution du taux d'attaque (Figure 2).

\section{Sur les essences de la Réserve de Faune de Togodo}

Les taux d'infestation de termites sur les 3 essences forestières varient d'une essence à une autre. Le taux moyen d'infestation est de 80,00 \pm 3,56\% pour Antiaris africana (Figure 4). Le pourcentage des pieds sains avec un système racinaire infesté est de $21,22 \%$. Les pieds portant les galeries et des placages sont respectivement de $44,44 \%$ et $2,22 \%$. Le nombre de pieds présentant une attaque dans le bois est évalué à 12, 22\% (Figure 5). En ce qui concerne Pterocarpus erinaceus, le taux moyen d'infestation est de $67,78 \pm 17,47 \%$ dont 4,44\% d'attaques de type PIV. Il constitue 1'essence ayant enregistré le moins d'attaques (Figure 4). Triplochiton scleroxylon est l'essence ayant enregistré le plus fort taux d'infestation $(97,78 \pm 0,99 \%)$ dont $31,11 \%$ d'attaque directe dans le bois (Figure 4). 


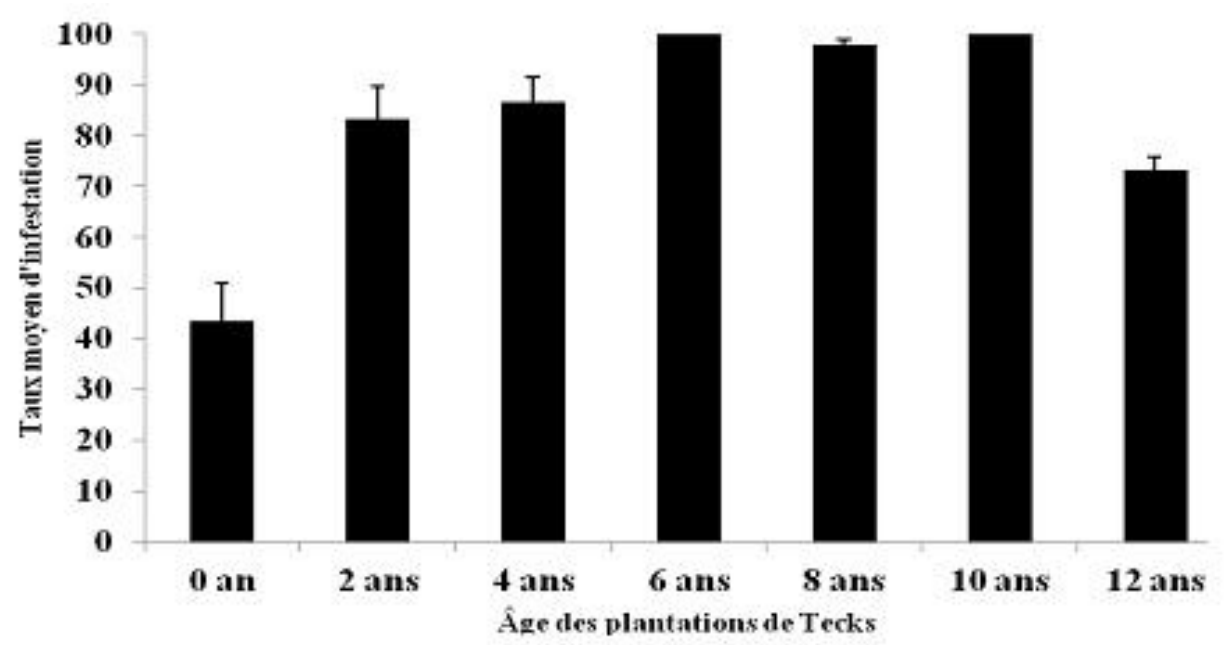

Figure 2 : Taux moyen d'infestation des termites dans les plantations de tecks

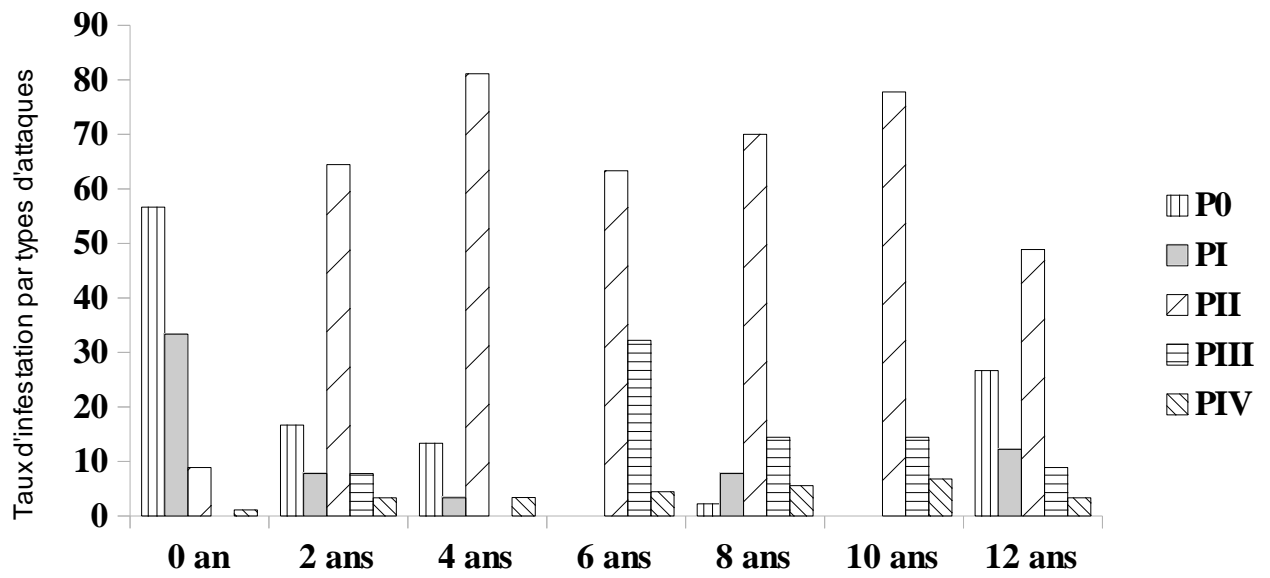

Figure 3 : Taux des types d'infestation par âge dans les plantations de tecks $\mathrm{P} 0=$ Arbre sain avec absence de termites, $\mathrm{PI}=$ Arbre sain avec présence de termites dans le sol, PII = Présence de galerie sur le tronc avec ou sans termites, PIII = Tronc d'arbre partiellement ou totalement couvert de placages de termites, sur au moins 2,5 $\mathrm{m}$ de hauteur, $\mathrm{PIV}=$ Arbre attaqué par les termites jusqu'à l'aubier. 


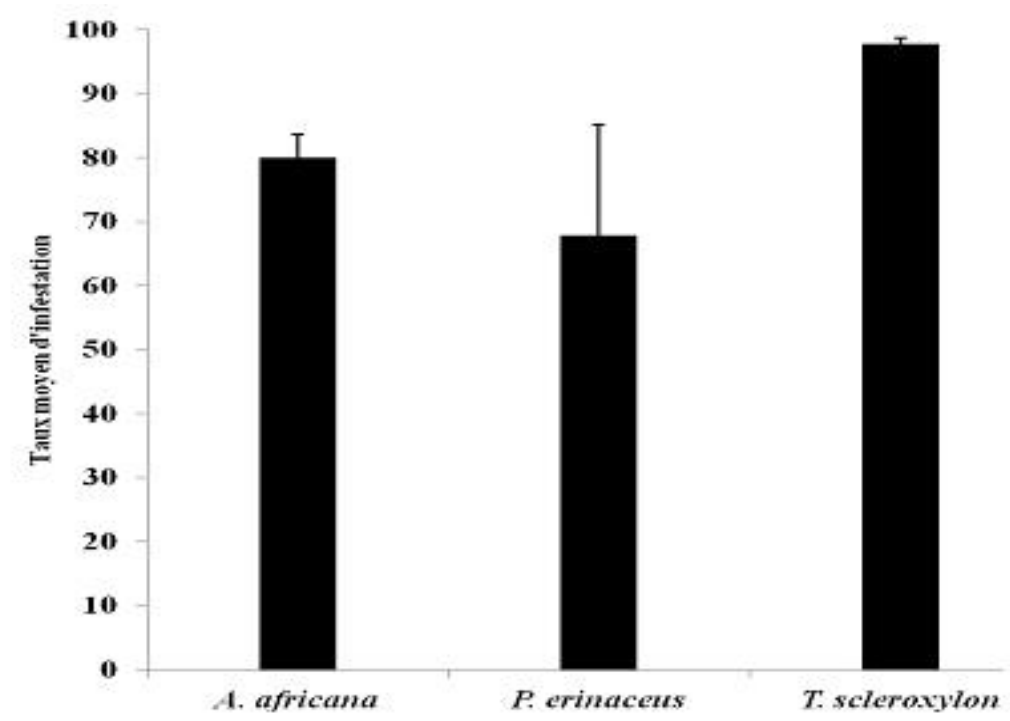

Figure 4 : Taux moyen d'infestation des termites sur les essences de forêt

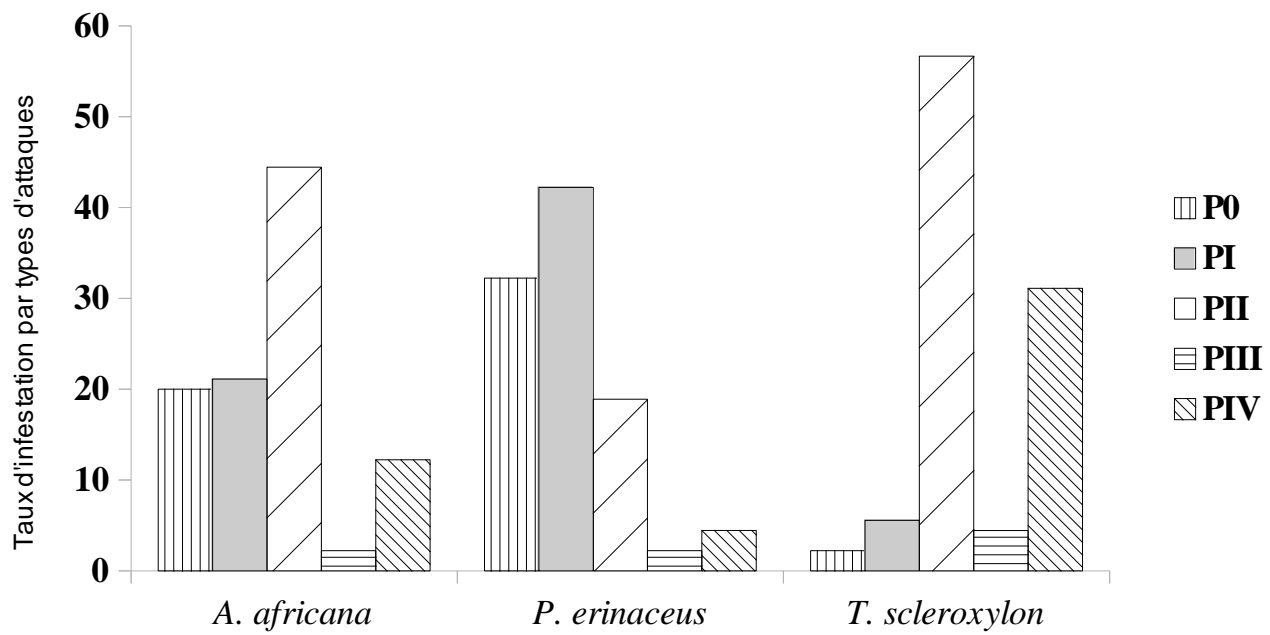

Figure 5 : Taux des types d'infestation par essence forestière

$\mathrm{P} 0=$ Arbre sain avec absence de termites, $\mathrm{PI}=$ Arbre sain avec présence de termites dans le sol, PII = Présence de galerie sur le tronc avec ou sans termites, PIII = Tronc d'arbre partiellement ou totalement couvert de placages de termites, sur au moins 2,5 $\mathrm{m}$ de hauteur, $\mathrm{PIV}=$ Arbre attaqué par les termites jusqu'à l'aubier.

\section{Qualification des infestations de termites}

Sur la base des indices d'infestation obtenus, le taux d'infestation dans les plantations de teck de 0 an est qualifié de faible alors qu'il est qualifié de fort dans les tecks de 2 et 4 ans, et de très fort dans les 
plantations de 6,8 et 10 ans. Ce taux est classé de moyen dans la plantation de teck de 12 ans. Les analyses statistiques montrent que les 4 niveaux d'infestations observés sont significativement différents (Tableau 3).

Les infestations qualifiées de moyen sur les 3 essences de la forêt ne montrent pas de différence statistiquement significative (Tableau 4).

Tableau 3: Qualification des indices d'infestation dans les plantations de tecks

\begin{tabular}{ccc}
\hline Tecks/Ages & Indice des infestations (\%) & Qualification des infestations \\
\hline 0 an & $12,79 \pm 2,8 \mathrm{a}$ & FAIBLE \\
2 ans & $40,03 \pm 0,7 \mathrm{~b}$ & FORT \\
4 ans & $41,41 \pm 1,8 \mathrm{~b}$ & FORT \\
6 ans & $55,88 \pm 2,4 \mathrm{c}$ & TRES FORT \\
8 ans & $50,27 \pm 3,3 \mathrm{c}$ & TRES FORT \\
10 ans & $50,62 \pm 0,6 \mathrm{c}$ & TRES FORT \\
12 ans & $34,20 \pm 0,5 \mathrm{~d}$ & MOYEN
\end{tabular}

Test statistique $: \mathrm{F}=71,398 ; \mathrm{df}=6 ; \mathrm{P}<0,001$

Dans chaque colonne, les moyennes portant les mêmes lettres ne sont pas statistiquement différentes au seuil de 5\% selon le test de StudentNewman-Keuls.

Tableau 4 : Classification des indices d'infestation dans les peuplements des essences de forêt

\begin{tabular}{ccr}
\hline Essences forestières & Indice des infestations (\%) & Qualification des in \\
\hline A. africana & $29,30 \pm 4,5 \mathrm{a}$ & MOYEN \\
P. erinaceus & $21,71 \pm 1,2 \mathrm{a}$ & MOYEN \\
T. scleroxylon & $33,40 \pm 1,1 \mathrm{a}$ & MOYEN
\end{tabular}

Test statistique : $\mathrm{F}=9,398 ; \mathrm{df}=2 ; \mathrm{P}=0,961$

Dans chaque colonne, les moyennes portant les mêmes lettres ne sont pas statistiquement différentes au seuil de 5\% selon le test de StudentNewman-Keuls.

\section{Analyse qualitative : modes d'attaque des différentes espèces de termites}

Les modes d'infestation des termites sont très variables selon les espèces rencontrées. Chez certaines espèces, la présence des termites et/ou des nids n'était obligatoirement pas associée à des dégâts sur les pieds concernés. Chez d'autres par contre, la présence des termites est très souvent accompagnée de dégâts plus ou moins sévères. En général, les dégâts sont observés sur les racines et les troncs et dans le bois avec des manifestations caractéristiques propres à certaines espèces. 


\section{Microcerotermes parvus}

M. parvus a été rencontré dans les plantations de tecks de 0 et 2 ans, sur Antiaris africana et Pterocarpus erinaceus avec parfois la présence d'un nid arboricole. L'espèce a été rencontrée dans le sol notamment au niveau des racines. Plusieurs galeries (tunnels) et placages en relation avec le sol longent le tronc des arbres attaqués. Dans ces galeries circulent les termites. Les attaques observées se limitent à l'écorce des arbres.

\section{Amitermes evuncifer}

A. evuncifer a été rencontré dans des galeries en terres noires sur les troncs d'arbres et dans le bois d'Antiaris africana, de Pterocarpus erinaceus et de Triplochiton scleroxylon mais aussi sur certains pieds des tecks de 4 et 10 ans. Les attaques de cette espèce se font sur l'écorce et dans le bois de ces plantes à l'abri des galeries ou des placages. Les galeries partent en grande partie du sol et remontent vers les parties supérieures des arbres. Les attaques les plus fréquentes qui atteignent rapidement le bois et le cambium de l'arbre se font à la faveur des fissures ou de petits trous sur le tronc. Des nids d' $A$. evuncifer ont été trouvés à la base de certains arbres sur le sol près des racines. Les attaques de cette espèce sur $T$. scleroxylon provoquent un écoulement de sève.

\section{Ancistrotermes guineensis}

A. guineensis est présente dans toutes les plantations de tecks et dans les peuplements des 3 essences forestières. Les attaques de cette espèce sont localisées sur l'écorce du tronc, avec souvent de spectaculaires constructions de galeries et de placages argileux. Elle est considérée comme responsable de la dégradation de l'écorce et de la consommation des parties mortes des arbres.

\section{Microtermes lepidus}

Cette espèce comme, Ancistrotermes guineensis, est présente partout. Ses attaques se situent le plus souvent sur les racines des arbres. Les dégâts se traduisent essentiellement par la consommation des racines avec des galeries de pénétration de petites tailles. Ces dernières sont souvent rebouchées de terre.

\section{Macrotermes subhyalinus}

Cette espèce était uniquement présente dans la plantation de tecks de 10 ans. M. subhyalinus construit des nids autour des pieds de tecks, des galeries et placages sur les troncs. Des galeries ou des placages argileux mis 
à nu laissent voir des ouvriers de $M$. subhyalinus se déplacer activement sur les troncs des arbres.

\section{Pseudacanthotermes militaris et Odontotermes aff. erraticus}

Les attaques de ces deux espèces de termites champignonnistes se font les plus fréquemment à l'abri de placages qui peuvent recouvrir l'ensemble du tronc et se poursuivre vers les branches. Ces placages leur servent de protection pour attaquer la plante. Sous les placages, on observe des morsures avec des galeries de pénétration de petites moyennes au niveau de l'écorce qui peuvent atteindre l'aubier. Ces attaques se font de l'extérieur vers l'intérieur.

\section{Fulleritermes tenebricus et Nasutitermes arborum}

Ces espèces de termites construisent des nids arboricoles, en carton de couleur noire autour d'une branche d'arbre. De ces nids partent, de spectaculaires galerie-tunnels sur les troncs et le plus souvent pouvant atteindre le sol. La destruction de ces galerie-tunnels laisse entrevoir des perforations dans l'écore des arbres infestés qui peuvent atteindre l'aubier. Ces attaques se font de l'extérieur vers l'intérieur.

Pour les espèces d'Adaiphrotermes sp. et Basidentitermes potens, leur présence n'est pas associée à un dégât. Ces espèces ont été récoltées dans le sous sol aux alentours immédiats des arbres examinés.

La planche 1 montre les photos illustratives des différentes attaques des termites sur les pieds.

\section{Discussion}

Onze espèces de termites ont été inventoriées. La présence de certaines de ces espèces n'est pas très souvent associée aux dégâts. Les termites attaquent les arbres compte tenu de leur régime alimentaire principalement à base de cellulose et de leur besoin en eau. En Afrique tropicale, des travaux ont montré qu'en plus des Macrotermitinae (Mora et al., 1990 ; Wood et Pearce, 1991) et des Termitinae (Sands, 1973 ; Wood et al., 1980), les Nasutitermitinae (Pretorius et al., 1991 ; Mampouya, 1997) sont aussi responsables d'importants dégâts causés aux plantations et aux essences forestières et fruitières ainsi qu'aux plantes horticoles. Dans des travaux similaires, certains auteurs ont signalé des attaques de termites sur plusieurs plantes domestiquées : acacia, anacardier, cacaoyer, cassia, cocotier, citronnier, eucalyptus, goyavier, hévéa, mandarinier, manguier, oranger, palmier à huile (Renoux, 1997). Sur le campus universitaire de Lomé, Anani Kotoklo et al. (2010b) ont recensé plus de 20 espèces végétales attaquées par les termites. Le taux était de 92,40\%, avec 3,8\% de mortalité. Au Nigeria, sur le campus de l'Université d'Ife, des termites ont causé d'importants dégâts sur des arbres ornementaux et d'ombrage (Malako, 
1983). Au Sénégal, dans la région de Casamance, vingt-trois espèces de termites ont été inventoriées sur des arbres fruitiers et causent d'importants dégâts (Ndiaye et Han, 2002). En Inde, plus de 40 espèces de plantes ornementales, d'arbres fruitiers et d'essences forestières ont été attaquées par les termites (Parihar, 1981). Entre 1975 et 1977 sur le campus de Bangalore, 20 à $40 \%$ des plants de manguiers ont été attaqués par les termites (Veeresh et al., 1988).

D'après nos résultats, nous remarquons que les infestations sont essentiellement de type PII (présence de galerie sur le tronc avec ou sans termites) avec un taux de plus de $75 \%$. Ce taux élevé est dû au mode d'attaques des différentes espèces de termites rencontrés. En effet, la plupart des termites construisent des galeries et des placages de terre sur les troncs des plantes. Ces placages leur servent de protection pour attaquer la plante. Selon Akpesse et al. (2008), l'attaque des termites se fait de l'extérieur vers l'intérieur avec consommation successive du cortex externe de la tige, du parenchyme interne et du cambium de la tige. L'action délétère des termites sur les arbres observés se traduit par des impacts d'attaques de termites laissés sur ces arbres. Cependant, cette action peut se traduire autrement chez l'arbre par la baisse de l'activité photosynthétique due à la couverture du tronc de l'arbre par des placages terreux. Logan et El Bakri (1990) ont montré que la présence de galeries et de placages de récolte sur l'arbre peut réduire sa capacité photosynthétique et lui causer le stress ; source de futures attaques de termites.

Dans nos observations, le taux d'attaque dû aux termites est variable selon les arbres. Dans les plantations de tecks, le taux d'attaques augmente avec l'âge de la plantation sauf dans la plantation de 12 ans. Les dégâts observés dans les plantations de tecks vont de la dégradation de l'écorce, à la chute des feuilles et quelques rares fois à la mort des pieds. Cette variabilité d'attaque pourrait être liée à la teneur en extraits (tanins, flavonoïdes, lignanes, stilbènes terpénoïdes, tropolones, etc.) et en lignine dont dépend la durabilité naturelle du bois face aux attaques des champignons et des insectes. De plus chez le teck, plus de 40 composés extractibles ont été identifiés (Simatupang et Yamamoto, 1999). Ce sont essentiellement les quinones (naphthoquinones et anthraquinones), les terpénoïdes et d'autres composés dérivés du déhydronaphthol (tectol et déhydrotectol). Les anthraquinones (spécialement le tectoquinone), le lapachol et le désoxylapachol interviennent dans la résistance vis-à-vis des termites, ce qui ne semble pas être le cas du tectol et du déhydrotectol. Ces deux substances semblent jouer le rôle de répulsion des insectes. En comparant les taux d'attaques chez les 3 essences forestières étudiées, nous remarquons que $T$. scleroxylon est l'espèce la plus attaquée $(97,78 \%)$ suivi d'Antiaris africana (80,00\%) et de Pterocarpus erinaceus $(67,78 \%)$. Les dégâts sur $T$. 
scleroxylon sont généralement matérialisés par un creux comblé ou non par de la terre dans le tronc de l'arbre. Ceci pourrait s'expliquer par le fait que le bois de cet arbre est tendre et léger. De ce fait résiste moins aux mandibules des termites (Kasseney, 2007; Anani Kotoklo et al., 2010b). Le bois d'Antiaris africana n'est pas aussi un bois dure; il est sujet aux attaques de champignons (par ex. le bleuissement), et sensible aux termites et aux térébrants du bois sec; l'aubier est sujet d'attaques de bostryches (Bosu et Krampah, 2005). Ceci pourrait expliquer le taux d'attaques élevé observé. Pour ce qui est de Pterocarpus erinaceus, son bois est plus ou moins dur et rouge par conséquent résistant aux attaques de termites et corroborent les travaux de Aming et al. (1985) et Mitchell et al. (1987) sur la résistance de certains arbres face aux attaques de termites.

Plusieurs espèces de termites sont présentes sur une même essence. Ceci semble indiquer que certaines essences seraient plus appétantes que d'autres. Roy-Noël (1974), Ndiaye et Han (2002) et Anani Kotoklo et al. (2010b) ont fait des observations similaires et ont montré que plusieurs espèces de termites se retrouvant sur les mêmes pieds d'arbres peuvent utiliser la même partie du végétal et entrer ainsi en compétition ou exploiter des régions anatomiques différentes du végétal.

La proportion de $100 \%$ d'arbres infestés par les termites dans les plantations de 6 et 10 ans pourrait être liée à la nature du sol (sol rouge ferralitique) qui favoriserait l'installation des termitières, sources des infestations des plantes. Thakur et al. (1989) et Anani Kotoklo et al. (2010b) ont observé que l'incidence et l'intensité des attaques des termites varient suivant l'espèce, la localité et les conditions édaphiques.

Des 11 espèces inventoriées, Amitermes evuncifer, Ancistrotermes guineensis et Microtermes lepidus sont les ravageurs majeurs de plants. Ces espèces sont citées par Folwer et Forti (1990), Pearce et al. (1995), Anani Kotoklo, (2007), Akpesse et al. (2008) et Coulibaly et al. (2014) comme responsables des dégâts importants sur les cultures et sur les arbres.

\section{Conclusion}

Différentes espèces de termites s'attaquent aux arbres. Cette étude a révélé que parmi les 11 espèces rencontrées, seules 2 ne se retrouvaient pas sur les pieds des arbres. Ainsi, ont été identifiées comme termites responsables des attaques de ces arbres Amitermes evuncifer, Microtermes lepidus et Ancistrotermes guineensis. Ces trois espèces de termite sont celles qui provoquent des dégâts importants. Les plantations de tecks de 6,8 et 10 ans sont les plus attaquées. Il en est de même pour Triplochiton scleroxylon parmi les essences forestières. Les modes d'attaque des termites sont très variables selon les espèces et portent généralement sur la consommation des écorces et de l'aubier, la construction des galeries et des placages sur les 
troncs. Les dommages provoqués par les termites sur d'autres arbres d'importances économiques et sur les cultures méritent d'être étudiés de manière exhaustive afin d'estimer les dégâts causés par les termites au Togo. Des méthodes de lutte adéquates contre ces insectes déprédateurs doivent être entreprises pour un bon développement de ces arbres.

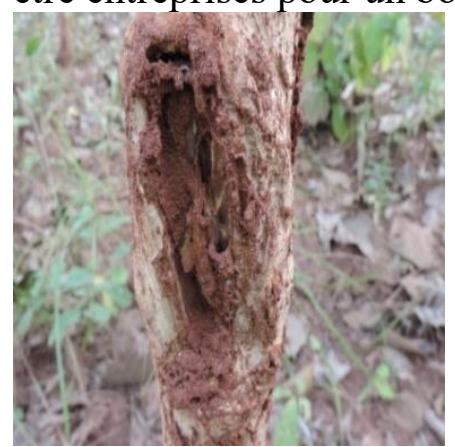

Photo a : Pied de teck dont l'écorce et une partie du bois détruis et remplacés par de la terre

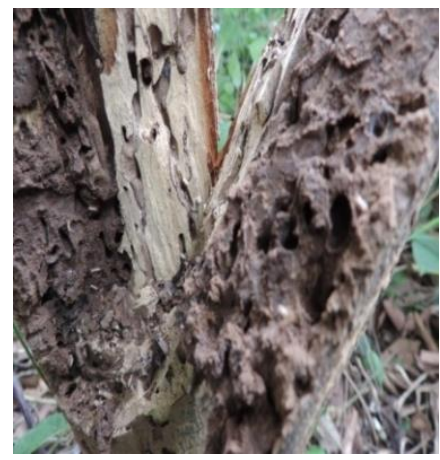

Photo c: Bois attaqués et détruits par Amitermes evuncifer

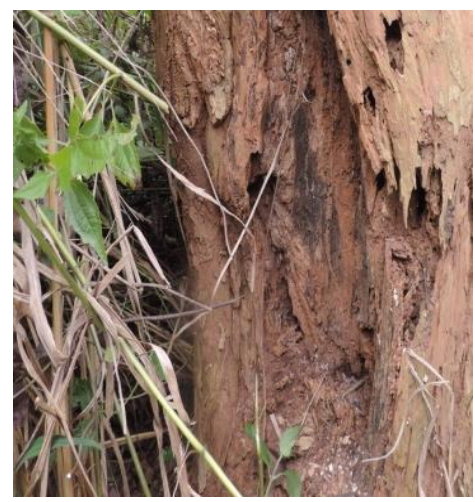

Photo e : Pied attaqué par Amitermes evuncifer avec destruction du bois

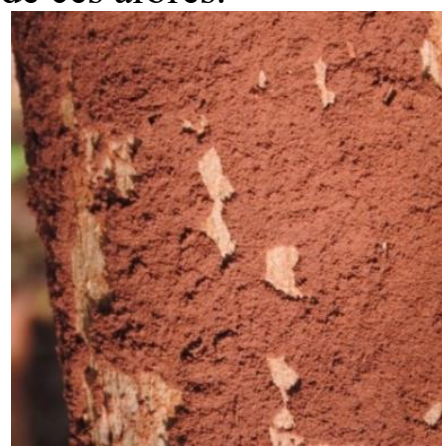

Photo b: Pied de teck attaqué par Ancistrotermes guineensis et dont la partie détruite est remplacée par de la terre

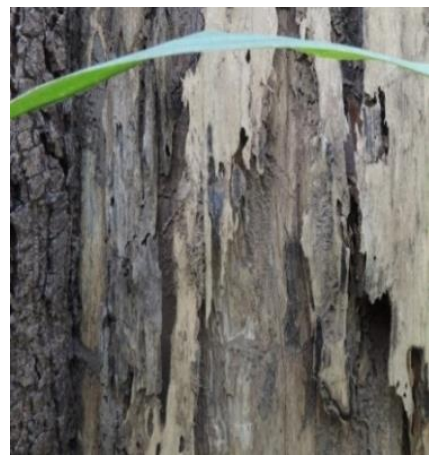

Photo $\mathbf{d}$ : Ecorce et bois détruits á I'intérieur desquels, il y a des individus d'Ancistrotermes guineensis

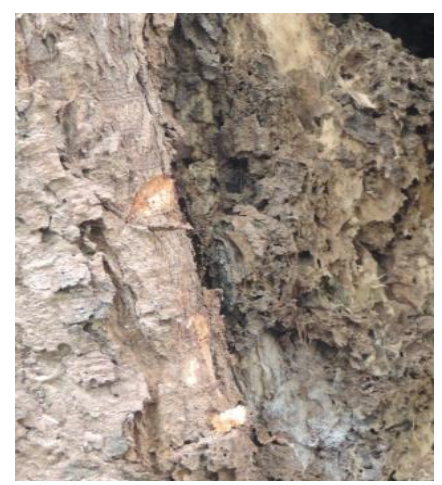

Photo f: Creux dans le tronc avec présence des meules à champignons d'Ancistrotermes guineensis 
Planche 1. Les différentes attaques observées sur les pieds des différentes essences

\section{References:}

Adjonou, K. (2011). Structure et indicateurs biologiques de gestion durable des reliques de forêts sèches du Togo. Thèse de Doctorat Unique. Université de Lomé, Togo, 185p.

Akpesse, A.A., Kouassi, P.K., Tano, Y. et Lepage, M. (2008). Impact des termites dans les champs paysans de riz et de maïs en savane subsoudanienne (Booro-Borotou, Côte-d'Ivoire). Sciences \& Nature 5 (2): 121131.

Aléné, D.C., Messi, J. et Quilici, S. (2006). Influence de l'ombrage sur la sensibilité des plantules de Ricinodendron heudelotii (Baill.) aux attaques de Diclidophlebia xuani en milieu naturel au Cameroun. Fruits, 61: 273-280.

Ali, N. (2009). Pterocarpus erinaceus (Fabaceae) dans la Région Centrale du Togo: Usages et impacts de l'exploitation sur la distribution spatiale et caractéristiques structurales. Mémoire de Technicien Supérieur Agricole de l'INFA de Tové, $43 \mathrm{p}$.

Aming, P.W., Singh, K.N., Dwivedi, S.L. and Rao, V.R. (1985). Resistance to the jassid (Empoasca kerri Pruthi), thrips (Franklinela schultzei (Trybom)) and termites (Odontotermes sp.) in groundnut (Arachis hypogea L.) Peanut Science 12: 58-60.

Anani Kotoklo, E. (2007). Inventaire des termites et évaluation de leurs dégâts dans deux champs de cannes à sucre au sud du Togo. Thèse de Doctorat, Université de Lomé, p. 231.

Anani Kotoklo, E., Kasseney, B.D., Nyamador, W., Kétoh, G.K. et Glitho, I.A. (2010). Attaques des arbres par les termites sur le campus de l'Université de Lomé (Togo). International Journal of Biology and Chemical Sciences 4 (1): 61-68.

Aubréville, A. (1959). La flore forestière de la Côte d'Ivoire. Deuxième édition révisée. Tome premier. No 15. Centre Technique Forestier Tropical, Nogent-sur-Marne, France. 369 pp.

Bhat, K.M.M. (2000). Qualité des bois de teck issus de plantations tropicales aménagées avec une mention particulière aux plantations indiennes. Bois et Forêts des Tropiques 263: 6-16.

Bosu, P.P. and Krampah, E. (2005). Antiaris toxicaria Lesch. In: Louppe, D., Oteng-Amoako, A.A. \& Brink, M. (Editors). PROTA (Plant Resources of Tropical Africa / Ressources végétales de l'Afrique tropicale), Wageningen, Netherlands. http://uses.plantnet-project.org/fr/Antiaris_toxicaria_(PROTA). Consulté le 9 novembre 2015.

Coulibaly, T., Akpesse, A.M., Yapi, A., Zirihi, G.N. et Kouassi, K.P. (2014). Dégâts des termites dans les pépinières de manguiers du nord de la Côte 
d'Ivoire (Korhogo) et essai de lutte par utilisation d'extraits aqueux de plantes. Journal of Animal \&Plant Sciences 22 (3): 3455-3468.

Cuny, P., Sanogo, S. et Sommer, N. (1997). Arbres du domaine soudanien. Leurs usages et leur multiplication. IER, Sikasso, Mali \& Intercoopération, Bern, Switzerland. 122 p.

Derwisch, S., Schwendenmann, L., Olschewski, R. and Hölscher, D. (2009). Estimation and economic evaluation of aboveground carbon storage of Tectona grandis plantations in Western Panama. New Forests 37: 227-240.

Duvall, C.S. (2008). Pterocarpus erinaceus Poir. In: Louppe, D., OtengAmoako, A.A. \& Brink, M. (Editors). PROTA (Plant Resources of Tropical Africa / Ressources végétales de l'Afrique tropicale), Wageningen, Netherlands. http://uses.plantnet-project.org/fr/Antiaris_toxicaria_(PROTA). Consulté le 9 novembre 2015.

FAO/PNUE, (1972). Développement des ressources forestières. Togo : Inventaire pédologique de certains secteurs du sud-Togo. Rome, 160 p.

Fofana, I.J., Ofori, D., Poitel, M. and Verhaegen, D. (2009). Diversity and genetic structure of teak (Tectona grandis L.f) in its natural range using DNA microsatellite markers. New Forests 37: 175-195.

Folwer, H.G. and Forti, L.C. (1990). Status and prospects of Termite problems and control in Brazil. Sociobiology 17 (1): 156-176.

Ganglo, J.C. (1999). Phytosociologie de la végétation naturelle de sous-bois, écologie et productivité des plantations de teck (Tectona grandis L.f.) du sud et du centre Bénin. Thèse de doctoract, Université libre de Bruxelles, Belgique, 366 pp.

Gbenyedji, J.N.B.K. (2009). Inventaire des dégâts des termites sur le bois stocké au sud du Togo. Mémoire de DEA, Université de Lomé (Togo) p. 67. Gorel, A. (2012). Étude de l'origine des populations d'Ayous (Triplochiton scleroxylon K. SCHUM) dans les forêts du sud-est du Cameroun. Mémoire de Master, Université de Liège, 87 p.

Kasseney, B.D. (2007). Diversité spécifique et dégâts des termites sur le campus de Lomé. Mémoire de DEA. Université de Lomé (Togo) p. 59

Logan, J.W.M. and El Bakri, A. (1990). Termite damage to date palms (Phoenix dactylifera L.) in northern Sudan with particular reference to the Dongola District. Tropical Sciences 30: 95-108.

Logan, J.W.M., Cowie, R.H. and Wood, T.J (1990). Termite (Isoptera) control in agriculture and forestry non-chemical methods: a review. Bulletin of Entomology Research 80: 309-330.

Louppe, D., Koua, M. et Coulibaly, A. (1994). Tarifs de cubage pour Pterocarpus erinaceus Poir. en Forêt de Badénou (Nord Côte d'Ivoire). IDEFOR/CIRAD-Forêt, Abidjan, Côte d'Ivoire $30 \mathrm{p}$.

Louppe, D. (2005). Tectona grandis L.f. In: Louppe, D., Oteng-Amoako, A.A. \& Brink, M. (Editors). PROTA (Plant Resources of Tropical Africa / 
Ressources végétales de l'Afrique tropicale), Wageningen, Netherlands. Consulté le 6 janvier 2016.

Malako, S.L.O. (1983). Economic importance of termites. Six cases studies in Nigeria and Ghana. The Nigeria Field 47(4): 222-230.

Mampouya, D. (1997). Les populations de termites d'une culture de canne à sucre irriguée dans un écosystème sahélien, Le fipronil dans une lutte ciblée contre les termites. Thèse d'Université, Université Paris XII- Val de Marne. $151 \mathrm{p}$.

Mitchell, M.R., Gwaze, D.P. and Stewart, H.T.L. (1987). The survival and early growth of Australian tree species in Zimbabwe, East African. Agricultural and Forestry Journal 54, 251-259.

Mora, P., Rouland, C., Dibangou, V. and Renoux, J. (1990). Damage caused by the recent infestation of the sugar cane fields by the fungus-growing termite Pseudacanthotermes spiniger, In: Veeresh $G K$, Mallik B, Viraktamath CA (Eds), Social Insects and the Environment. 604-605, Oxford IBH, New Delhi, India.

Ndiaye, A.B. et Han, S.H. (2002). Attaques des arbres fruitiers par les termites en Casamance (Sénégal) (Isoptera). Bulletin de la Société Entomologique de France 107 (2): 193-199.

Parihar, D.R. (1981). Some observations on distribution and pest status of termites attacking forestry plantations in the Rajasthan desert. Indian Journal of Forestry 4: 22-25.

Pearce, M.J., Logan, J.W.M. and Tiben, A. (1995). Termites (Isoptera) from the Darfur region of the Sudan with comments on their pest status. Journal of Arid Environment 30: 197-206.

Pretorius, M.W., Van Ark, H. and Mohr, J.D. (1991). Preliminary moundfumigation trials for the control of Trinervitermes trinervoides colonies (Isoptera: Termitidae). Phytophylactica 23: 89-90.

Rance, W. and Monteuuis, O. (2004). Teak in Tanzania. Bois et Forêts des Tropiques 279: 5-10.

Renoux, J. (1997). Les termites et l'homme. Laboratoire EBENA- Univ Paris XII pp 133-146.

Roy-Noë,1 J. (1974). Recherches sur l'écologie des Isoptères de la Presqu'île du Cap vert (Sénégal). Les espèces et leur écologie. Bulletin de l'IFAN 44(12): 115-146.

Sands, W.A. (1973). Termites as pests of tropical food crops. Pans 19 (2): 167-177.

Simatupang, H.M. and Yamamoto, K. (1999). Properties of teakwood (Tectona grandis L.f) as influenced by wood extractives and its importance for tree breeding. Regional seminar on site technology and productivity of teak plantations, Thailand, 141. 
Thakur, M.L., Kumar, S., Negi, A. and Rawat, D.S. (1989). Chemical control of termites in Eucalyptus Hybrid. Indian Forester 56: 733-742.

Von Maydell, H.J. (1983). Arbres et arbustes du Sahel. Leurs Caractéristiques et leurs utilisations. Eschnorn, GTZ. 531 p.

Veeresh, G.K., Rajagopal, D. and Kumar, N.G. (1988). Management of termites in mango orchard. Acta Horticulturae 231: 633-636.

Wood, T.G., Johnson, R.A. and Ohiagu, C.E. (1980). Termite damage and crop loss in Nigeria a review of termite (Isoptera) damage to maize and estimation of damage, loss in yield and termite (Microtermes) abundance at Makwa. Tropical Pest Management 26 (3): 241-253.

Wood, T.G. and Pearce, M.J. (1991). Termites in Africa: The environmental impact of control measures and damage to crops, trees, rangeland and rural buildings. Sociobiology 19 (1): 221-234. 